\title{
Comparaison de techniques mixtes pour le calcul de courants de Foucault en géométrie axisymétrique
}

\author{
L. Pichon, M. Ayoub et A. Razek \\ Laboratoire de Génie Electrique de Paris URA 127 C.N.R.S., Ecole Supérieure d'Electricité, Universités \\ Paris VI et Paris XI, Plateau du Moulon, 91192 Gif-sur-Yvette, France
}

(Reçu le 3 février 1989, révisé le 6 juillet 1989, accepté le 20 juillet 1989)

\begin{abstract}
Résumé. - Nous présentons et comparons dans cet article deux types de méthodes mixtes pour le calcul des courants de Foucault lors de la modélisation de systèmes électromagnétiques présentant une symétrie de révolution. Dans ces deux techniques, les éléments finis sont réservés aux parties massives du dispositif tandis que l'espace environnant est considéré ou bien par la méthode des éléments de frontière ou bien par le développement d'une solution analytique. Après avoir exposé les formulations employées et explicité les couplages réalisés, nous appliquons ces méthodes au calcul des courants de Foucault et comparons nos résultats numériques à ceux de l'expérience.
\end{abstract}

\begin{abstract}
In this paper two hybrid methods for eddy currents problems in axisymmetric electromagnetic systems are presented and compared. In these two techniques finite elements are kept for bounded regions of the installation while the free space is considered either with a boundary integral equation method or an analytical solution. We present the formulations of the methods and the combinations performed. These techniques are used for eddy currents calculation and numerical results are compared with experimental ones.
\end{abstract}

\section{Introduction.}

La méthode des éléments finis est maintenant reconnue comme un outil efficace pour le calcul des champs dans les systèmes électromagnétiques. Les récents développements de cette technique sont d'ailleurs plutôt relatifs à l'augmentation de la puissance des calculateurs qu'à l'analyse numérique qui se rapporte à la méthode. D'autres méthodes de calcul bénéficient également de l'extension des moyens informatiques en électrotechnique ; certaines sont depuis longtemps utilisées, il s'agit par exemple des transformations fonctionnelles de type Fourier ou Hankel ou des méthodes basées sur le développement en série des solutions, d'autres sont plus récentes comme par exemple la méthode des éléments de frontière $[1,2]$. Les premières ne sont utilisables que dans le cas de configurations géométriques simples ou supposent une périodicité dans la structure du système étudié. Elles peuvent néanmoins donner des résultats quantitatifs importants pour le comportement au voisinage d'une singularité ou le comportement à l'infini. La seconde transforme les équations aux dérivées partielles dans un domaine en une équation intégrale à la frontière de ce domaine : seule la discrétisation de la frontière est nécessaire pour résoudre alors le problème.

$\mathrm{Si}$ elles ne peuvent concurrencer la méthode des éléments finis là où celle-ci donne entière satisfaction en particulier en présence de non-linéarités, les techniques citées précédemment peuvent parfois fournir une alternative intéressante. Un obstacle souvent rencontré par les éléments finis dans beaucoup d'applications est la prise en compte des conditions aux limites existant à l'infini : l'utilisation d'un maillage pour approcher le problème réel demande alors un nombre de nœuds important et préjudiciable au temps de calcul. Dans une telle situation il semble naturel d'associer aux éléments finis une autre technique de calcul afin d'effectuer une modélisation fidèle d'un dispositif avec un coût raisonnable. C'est un choix souvent judicieux dans le cas bidimensionnel [3-6, 11] mais qui devient alors crucial en trois dimensions [1, 7].

Nous effectuons dans ce qui suit deux types de couplage dans le cadre d'une géométrie axisymétri- 
que : 1) une combinaison éléments finis/éléments de frontière ; 2) une combinaison éléments finis/développement analytique. Dans les deux cas la méthode des éléments finis est utilisée pour résoudre l'équation de diffusion alors que la méthode des éléments de frontière et le développement analytique sont employés pour résoudre l'équation de Laplace. Après avoir rappelé les formulations mathématiques utilisées, nous comparons ces deux techniques sur un même dispositif et mettons en évidence les avantages qu'elles possèdent sur une résolution par éléments finis classiques. Nous utilisons les deux couplages réalisés pour le calcul de courants de Foucault dans le cas d'une installation industrielle : les éléments finis modélisent les parties massives de l'installation tandis que l'espace environnant est considéré ou bien grâce aux éléments de frontière ou bien grâce à un développement analytique.

\section{Equations de base.}

Dans le cas d'une symétrie de révolution le potentiel vecteur magnétique et le vecteur densité de courant n'ont qu'une composante dans la direction azimutale que nous noterons $A$ et $J$ respectivement. Nous nous intéressons au problème suivant :

Soit $\Omega$ un domaine borné du plan susceptible de contenir des courants induits inconnus ou bien des courants sources et dans lequel $A$ est régi par l'équation générale de diffusion :

$$
\frac{\partial}{\partial z}\left\{\nu \frac{\partial A}{\partial z}\right\}+\frac{\partial}{\partial r}\left\{\frac{\nu}{r} \frac{\partial}{\partial r}(r A)\right\}-\sigma \frac{\partial A}{\partial t}+J=0
$$

où $\nu$ est la reluctivité magnétique et $\sigma$ la conductivité électrique.

Soit $\boldsymbol{\Omega}_{\mathrm{c}}$ le domaine complémentaire (non borné) de $\Omega$, linéaire $\left(\nu=\nu_{0}\right)$, non conducteur, et dans lequel $A$ est gouverné par l'équation de Laplace :

$$
\frac{\partial^{2} A}{\partial^{2} z}+\frac{\partial^{2} A}{\partial^{2} r}+\frac{1}{r} \frac{\partial A}{\partial r}-\frac{A}{r^{2}}=0
$$

Soit $\Gamma$ la frontière commune entre $\Omega$ et $\Omega_{\mathrm{c}}$.

Afin de simplifier les expressions mathématiques et d'assurer plus facilement la continuité des grandeurs électromagnétiques nous envisagerons le changement de variable :

$$
A^{\prime}=r A \text {. }
$$

\section{La méthode des éléments finis.}

Si on recouvre le domaine $\boldsymbol{\Omega}$ par des éléments finis triangulaires d'ordre 1 , une formulation variationnelle de (1) et une approximation numérique de Galerkin conduisent à une équation matricielle du type :

$$
[S]\left[A^{\prime}\right]+[T]\left[\frac{\partial A^{\prime}}{\partial t}\right]-[F]+[B]\left[\frac{\nu}{r} \frac{\partial A^{\prime}}{\partial n}\right]_{\Gamma}=0
$$

où $[S]$ est la matrice de raideur (matrice carrée, bande, symétrique),

[T] est la matrice d'amortissement (matrice carrée, bande, symétrique),

$[F]$ est le vecteur source,

$[B]$ est une matrice où les seules lignes non nulles sont celles correspondant à un nœud situé sur $\Gamma$.

$\left[A^{\prime}\right],\left[\frac{\partial A^{\prime}}{\partial t}\right],\left[\frac{\nu}{r} \frac{\partial A^{\prime}}{\partial n}\right]_{\Gamma}$ sont respectivement les vecteurs des valeurs nodales de $A^{\prime}, \frac{\partial A^{\prime}}{\partial t},\left.\frac{\nu}{r} \frac{\partial A^{\prime}}{\partial n}\right|_{\Gamma}$ (n : normale intérieure à $\Omega$ ).

\section{La méthode des éléments de frontière.}

La solution de (2) dans $\Omega_{\mathrm{c}}$ admet une représentation en potentiel de simple couche :

$$
\begin{aligned}
A^{\prime}\left(r_{\mathrm{s}}, z_{\mathrm{s}}\right) & =r_{\mathrm{s}} A\left(r_{\mathrm{s}}, z_{\mathrm{s}}\right) \\
& =\int_{\Gamma} G\left(r, r_{\mathrm{s}}, z, z_{\mathrm{s}}\right) q(r, z) \mathrm{d} \gamma
\end{aligned}
$$

où $\nu_{0} q$ représente une distribution fictive de charges sur $\Gamma$.

$$
\begin{gathered}
G\left(r, r_{\mathrm{s}}, z, z_{\mathrm{s}}\right)=\frac{\sqrt{r r_{\mathrm{s}}}}{\pi k}\left[\left(1-\frac{k^{2}}{2}\right) K(k)-E(k)\right] \\
k^{2}=\frac{4 r r_{\mathrm{s}}}{\left(r+r_{\mathrm{s}}\right)+\left(z-z_{\mathrm{s}}\right)} .
\end{gathered}
$$

$K$ et $E$ sont les intégrales elliptiques complètes de $1^{\text {re }}$ et $2^{\mathrm{e}}$ espèce respectivement [8]. De (4) on tire l'expression de la dérivée normale sur $\Gamma$ (cf. annexe) :

$$
\frac{1}{r_{\mathrm{s}}} \frac{\partial A^{\prime}}{\partial n_{\mathrm{s}}}=\frac{q\left(r_{\mathrm{s}}, z_{\mathrm{s}}\right)}{2}+\int_{\Gamma} \frac{1}{r_{\mathrm{s}}} \frac{\partial G}{\partial n_{\mathrm{s}}}\left(r, r_{\mathrm{s}}, z, z_{\mathrm{s}}\right) q(r, z) \mathrm{d} \gamma
$$

où $\mathbf{n}_{\mathrm{s}}$ est la normale extérieure à $\boldsymbol{\Omega}_{\mathrm{c}}$.

Une formulation variationnelle de (4) et (6) ainsi qu'une approximation de Galerkin avec des fonctions de forme d'ordre 1 conduisent aux relations matricielles suivantes :

et

$$
\begin{gathered}
{[R]\left[A^{\prime}\right]_{\Gamma}=[G][q]_{\Gamma}} \\
{[R]\left[\frac{\nu_{0}}{r_{\mathrm{s}}} \frac{\partial A^{\prime}}{\partial n_{\mathrm{s}}}\right]_{\Gamma}=[H][q]_{\Gamma}}
\end{gathered}
$$

où toutes les matrices intervenant dans (7) dépendent de la géométrie et de la discrétisation de la frontière. $[R]$ est une matrice creuse. $[G]$ et $[H]$ sont des matrices carrées pleines : le calcul de leurs éléments nécessite des intégrations doubles sur la frontière ; nous avons opté pour l'approximation numérique suivante : 
- lorsque deux segments sont distincts on utilise la quadrature de Gauss la plus simple possible : celle à un seul point d'intégration ;

- lorsque deux segments sont confondus on utilise une double quadrature de Gauss à 2 et 3 points pour éviter toute singularité.

On tire de (7) une relation liant $A^{\prime}$ et $\frac{\nu_{0}}{r_{\mathrm{s}}} \frac{\partial A^{\prime}}{\partial n_{\mathrm{s}}}$ sur la frontière :

$$
\left[\frac{\nu_{0}}{r_{\mathrm{s}}} \frac{\partial A^{\prime}}{\partial n_{\mathrm{s}}}\right]_{\Gamma}=\left[R^{-1}\right][H]\left[G^{-1}\right][R]\left[A^{\prime}\right]_{\Gamma} .
$$

\section{La solution analytique à l'extérieur d'un disque.}

Si la frontière $\Gamma$ représente la demi-circonférence d'un disque, la solution de (2) dans $\Omega_{\text {c }}$ peut alors se chercher sous la forme d'un développement en série. Le passage des coordonnées cylindriques aux coordonnées sphériques conduit à effectuer les changements de variables :

$$
r=\rho \sin \theta \quad \text { et } \quad z=\rho \cos \theta .
$$

On peut alors exprimer (2) sous la forme :

$$
\begin{array}{r}
\sin ^{2} \theta \frac{\partial}{\partial \rho}\left(\rho^{2} \frac{\partial A}{\partial \rho}\right)+\sin \theta \frac{\partial}{\partial \theta}\left(\sin \theta \frac{\partial A}{\partial \theta}\right)- \\
-A=0 .
\end{array}
$$

Si l'on cherche une solution de (9) à variables séparées la solution générale obtenue est alors [9] :

$$
A(\rho, \theta)=\sum_{n=1}^{+\infty} c_{n}\left(\frac{a}{\rho}\right)^{n+1} P_{n}^{1}(\cos \theta)
$$

où $a$ est le rayon du disque,

$\left[P_{n}^{1}\right]$ sont les fonctions de Legendre associées de $1^{\text {re }}$ espèce,

$\left[c_{n}\right]$ un ensemble de constantes réelles à déterminer.

Compte tenu du changement de variable on a :

$$
A^{\prime}(\rho, \theta)=r A(\rho, \theta)=\rho \sin \theta A(\rho, \theta) .
$$

Il devient possible à partir de (10) d'exprimer les dérivées partielles $\frac{\partial A^{\prime}}{\partial \rho}, \frac{\partial A^{\prime}}{\partial \theta}$, puis, compte tenu des relations entre coordonnées d'expliciter $\frac{\partial A^{\prime}}{\partial r}, \frac{\partial A^{\prime}}{\partial z}$ et donc $\frac{1}{r} \frac{\partial A^{\prime}}{\partial n}$. La discrétisation du contour $\Gamma$ nous permet de limiter le nombre de termes dans (10) ; en chacun des points de collocation on peut écrire :

$$
A_{i}^{\prime}=\sum_{n=1}^{M} r_{i} c_{n} P_{n}^{1}\left(\cos \theta_{i}\right) \quad i=1, \ldots, M
$$

où $M$ est le nombre de points de collocation sur $\Gamma$.
Les deux relations matricielles obtenues sont alors du type :

$$
\left[A^{\prime}\right]_{\Gamma}=[P][c] \text { et }\left[\frac{\nu_{0}}{r} \frac{\partial A^{\prime}}{\partial n}\right]_{\Gamma}=[Q][c]
$$

où les matrices intervenant dans (12) sont pleines et ne dépendent que de la position des points de discrétisation sur $\Gamma$.

En éliminant les valeurs de $[c]$ dans (12) il vient une relation analogue à $(8)$ :

$$
\left[\frac{\nu_{0}}{r} \frac{\partial A^{\prime}}{\partial n}\right]_{\Gamma}=[Q]\left[P^{-1}\right]\left[A^{\prime}\right]_{\Gamma}
$$

\section{Couplage des formulations.}

Le couplage de la formulation dans le domaine $\Omega$ avec celle dans le domaine extérieur $\Omega_{\mathrm{c}}$ s'effectue par. la continuité des quantités $A^{\prime}$ et $\frac{\nu}{r} \frac{\partial A^{\prime}}{\partial n}$ (composante tangentielle du champ magnétique) à travers $\Gamma$.

Les expressions (8) et (13) permettent, selon le couplage désiré de transformer le terme de frontière dans (3); il vient :

$$
[B]\left[\frac{\nu}{r} \frac{\partial A^{\prime}}{\partial n}\right]_{\Gamma}=\left[S_{\text {ext }}\right]\left[A^{\prime}\right]
$$

avec :

$$
\left[S_{\text {ext }}\right]=[B]\left[R^{-1}\right][H]\left[G^{-1}\right][R]
$$

dans le cas d'un couplage entre la méthode des éléments finis et la méthode des éléments de frontière et :

$$
\left[S_{\mathrm{ext}}\right]=[B][Q]\left[P^{-1}\right]
$$

dans le cas d'un couplage entre la méthode des éléments finis et la solution analytique.

$\left[S_{\text {ext }}\right]$ est appelée matrice de raideur extérieure.

En reportant (14) dans (3) on peut alors ajouter aux éléments d'une ligne de $[S]$ relative à un nœud situé sur $\Gamma$, les éléments de la ligne de $\left[S_{\text {ext }}\right]$ correspondant à ce même nœud ; (3) devient alors :

$$
\left[S_{\mathrm{c}}\right]\left[A^{\prime}\right]+[T]\left[\frac{\partial A^{\prime}}{\partial t}\right]-[F]=0
$$

où ne figurent plus que les valeurs nodales de $A^{\prime}$ et où la nouvelle matrice de raideur $\left[S_{\mathrm{c}} \mid\right.$ n'est plus symétrique.

Un schéma d'Euler implicite du $1^{\text {er }}$ ordre est utilisé pour résoudre (15).

\section{Résultats numériques et comparaison des coupla- ges.}

Nous comparons ici les résultats numériques obtenus par les deux types de couplages proposés : 
1) combinaison éléments finis-équations intégrales ;

2) combinaison éléments finis-développement analytique.

Nous avons choisi, comme exemple d'application de nos techniques couplées un dispositif qui soit de nature industrielle : il s'agit du système de chauffage par induction présenté par Al-Shaikhli et Hobson [10]; il consiste en une billette cylindrique en aluminium chauffée par une bobine inductrice parcourue par un courant de $600 \mathrm{~A}$ à la fréquence de $50 \mathrm{~Hz}$.

Les calculs sont effectués en négligeant les courants de Foucault dans la bobine excitatrice : on y résout ainsi l'équation de Poisson $(\sigma=0$ dans l'Eq. (1)) tandis que l'équation de diffusion homogène est utilisée pour la billette $(J=0$ dans l'Eq. (1)) ; l'espace environnant est régi par l'équation de Laplace.
Les figures 2 et 3 sont représentatives des couplages utilisés :

couplage 1 : la billette et l'inducteur sont discrétisés par éléments finis tandis qu'une équation intégrale de frontière qui s'appuie sur les parties massives prend en compte l'air environnant (Fig. 2) ;

couplage 2: il est nécessaire de discrétiser par éléments finis l'intérieur d'un demi-disque englobant la billette et l'inducteur. A l'extérieur un développement analytique est alors possible (Fig. 3).

On utilise la symétrie par rapport à l'axe $z=0$ pour réduire de moitié les domaines de résolution dans chaque cas.

Le calcul des valeurs du potentiel vecteur nous permet de déterminer la densité des courants induits dans la billette et donc la puissance dissipée par effet Joule. Nos résultats sont comparés à ceux de AlShaikhli et Hobson où les courants de surface ont été mesurés précisément avec des sondes de courant :

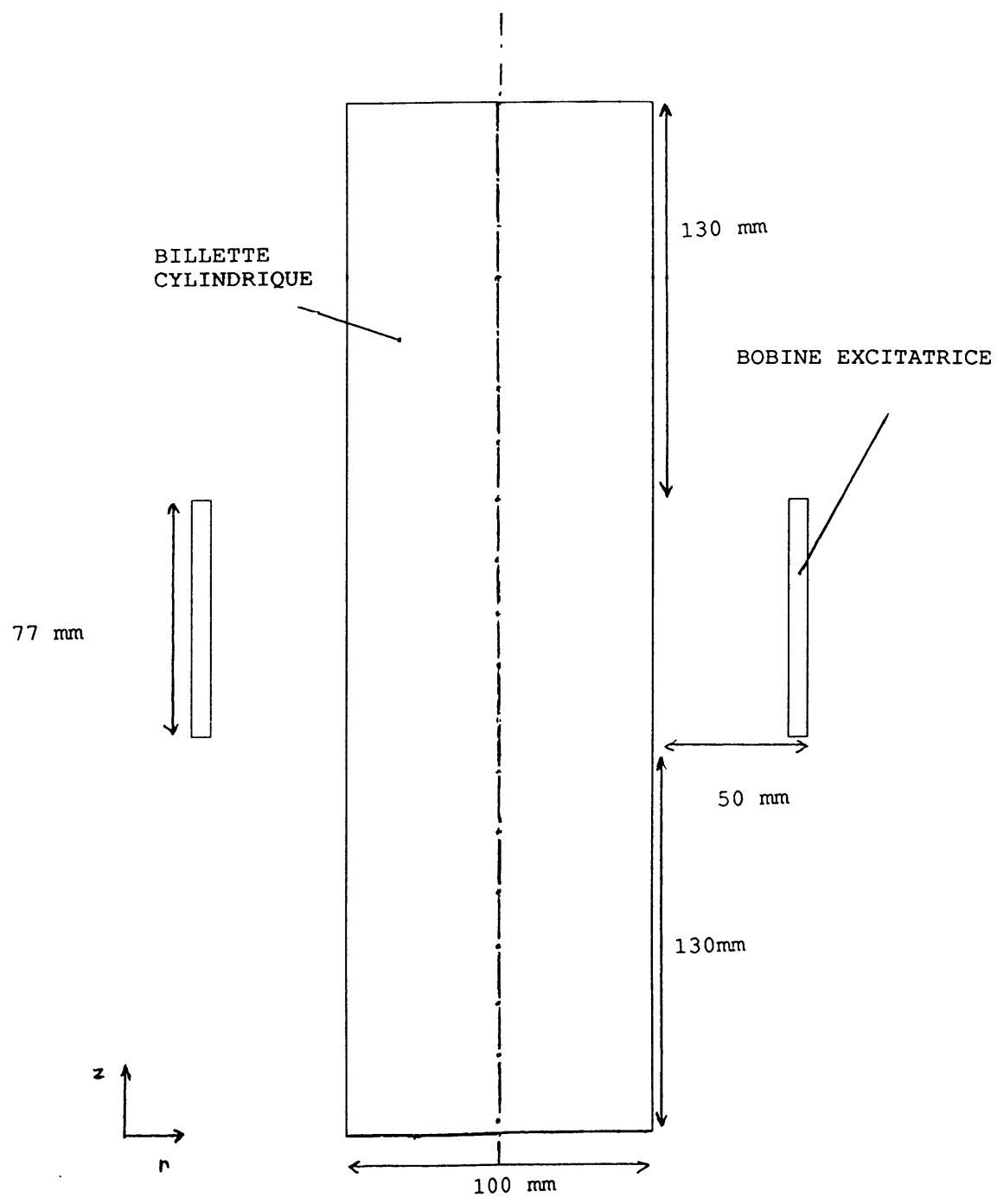

Fig. 1. - Dispositif étudié.

[Studied device.] 


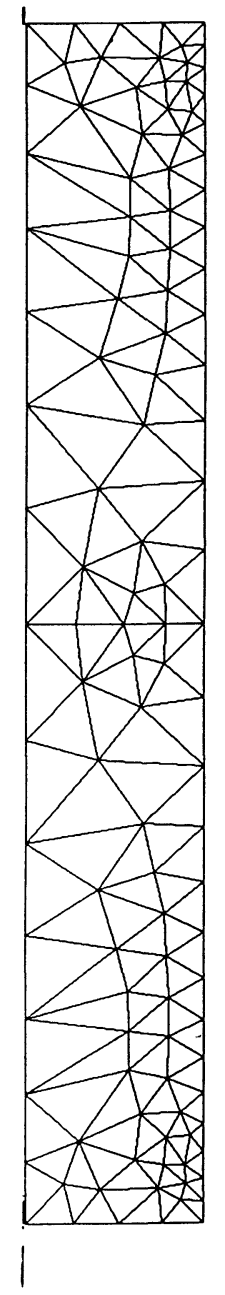

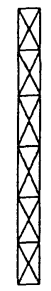

Fig. 2. - Maillage éléments finis pour la billette et la bobine (couplage 1 : éléments finis/équations intégrales).

[Finite element mesh for billet and coil (combination 1 : finite elements/boundary elements).]

les courbes de la figure 4 montrent la bonne concordance des résultats obtenus avec les résultats expérimentaux quel que soit le couplage effectué, l'erreur maximale étant de $5 \%$.

La figure 5 montre les lignes de flux obtenues dans la billette pour le couplage $n^{\circ} 1$ lorsque le courant dans la bobine inductrice est maximal. La figure 6 montre au même instant et pour un même nombre de lignes de flux les résultats dans le cas du couplage $\mathrm{n}^{\circ} 2$.

Pour les maillages choisis, il faut noter que le calcul de la matrice de rigidité extérieure pour le couplage $\mathrm{n}^{\circ} 1$ est plus coûteux que dans le couplage $\mathrm{n}^{\circ} 2$ à cause du plus grand nombre de nœuds sur la frontière et des intégrations numériques nécessaires. Globalement pourtant le couplage $n^{\circ} 2$ possède un temps de calcul deux fois plus important que celui du couplage $\mathrm{n}^{\circ} 1$. En effet dans le cas d'une méthode mixte la matrice $\left[S_{c}\right]$ qui apparaît dans $(15)$ possède une bande élargie. Dans le cas bidimensionnel qui

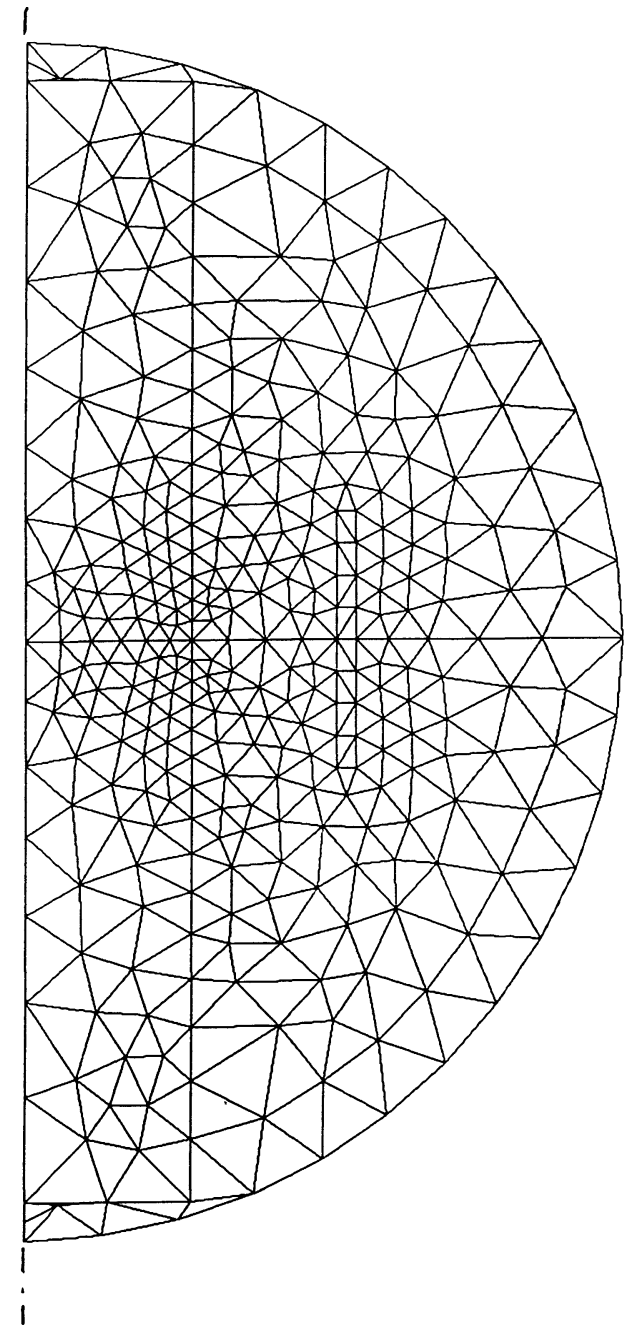

Fig. 3. - Maillage d'un demi-disque englobant la billette et la bobine (couplage 2 : éléments finis/solution analytique).

[Finite element mesh of half a disc containing billet and coil (combination 2 : finite elements/analytical solution).]

nous occupe, nous avons choisi de stocker la matrice $\left[S_{\mathrm{c}}\right]$ entièrement compte tenu du nombre d'inconnues relativement faible requis; sa dimension est plus importante dans le couplage $n^{\circ} 2$ puisque tout l'intérieur du demi-disque est discrétisé d'où un accroissement du temps de calcul.

Il nous a paru intéressant de comparer les deux techniques que nous avons mises en œuvre à une résolution classique par éléments finis. Le maillage utilisé est celui de la figure 7 . Les résultats numériques sont en bonne concordance avec ceux obtenus précédemment. Le temps d'exécution requis est sensiblement égal à celui nécessité par un couplage avec la méthode des équations intégrales de frontière. En revanche le nombre d'éléments stockés est d'environ $30 \%$ supérieur à cause du nombre de nœuds supplémentaires dans l'espace extérieur. 


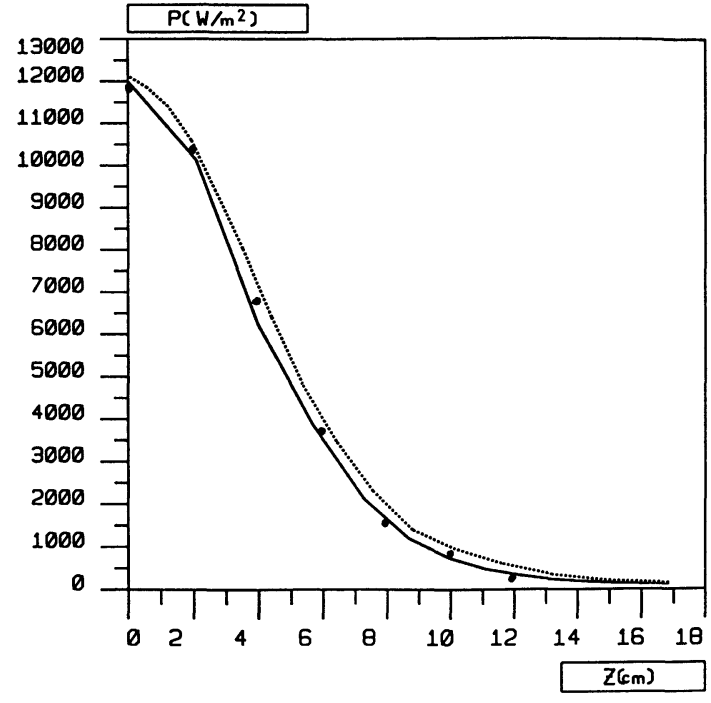

Fig. 4.-Densité surfacique de la puissance volumique dissipée dans une épaisseur de peau: valeurs calculées $(\longrightarrow)$ couplage $1,(---)$ couplage 2 ; valeurs mesurées $(\bullet)$.

[Surface density of volume dissipated power through the skin depth : computed values $(\longrightarrow$ ) combination 1 , $(---)$ combination 2 ; measured values $(\bullet)$.

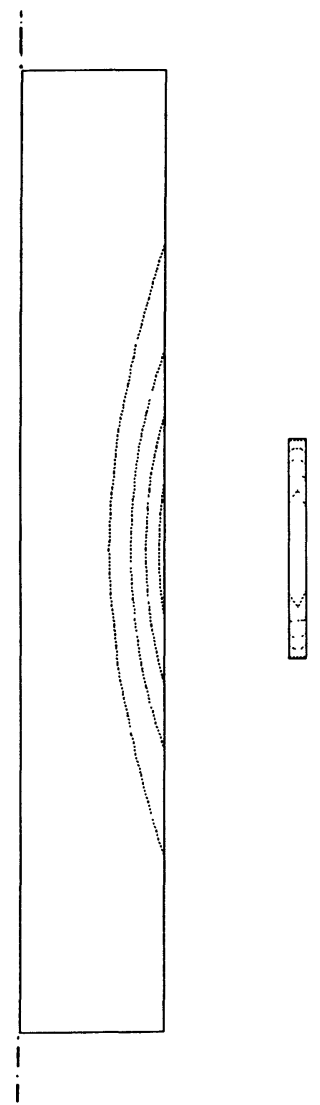

Fig. 5. - Equipotentielles vecteurs obtenues avec le couplage 1. $\Delta A^{\prime}=2 \times 10^{-5}$.

[Equipotential lines obtained with combination 1. $\Delta A^{\prime}=$ $2 \times 10^{-5}$.]

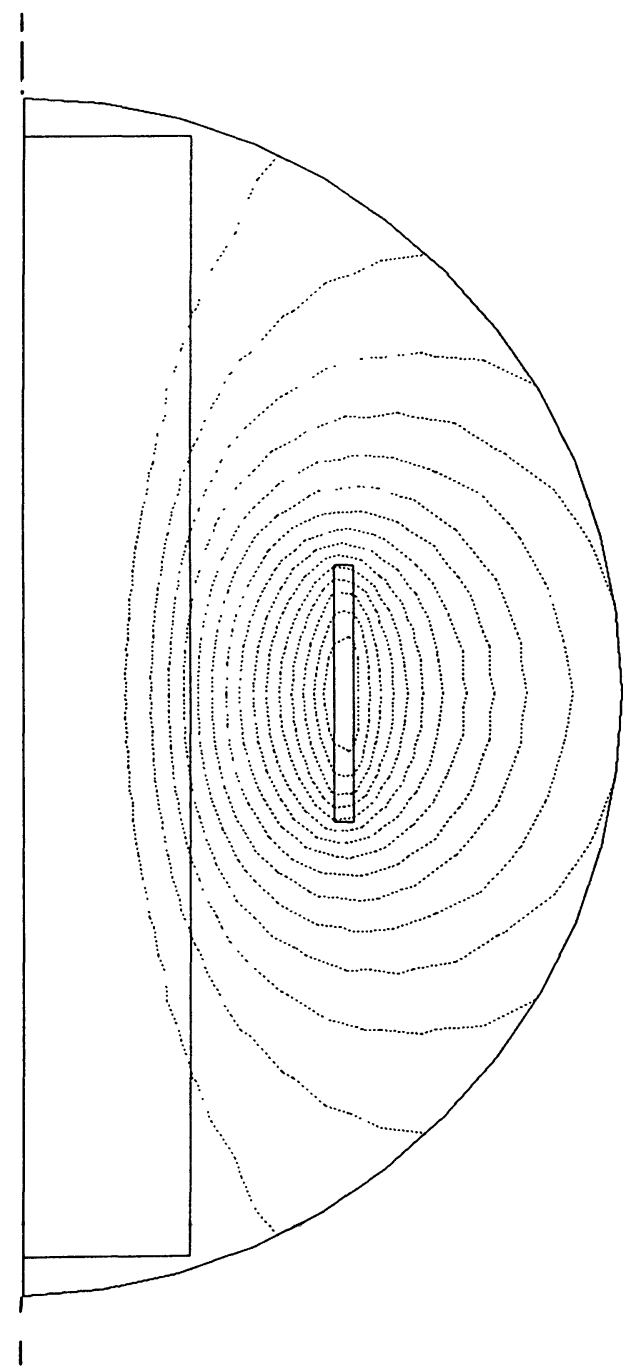

Fig. 6. - Equipotentielles vecteurs obtenues avec le couplage 2. $\Delta A^{\prime}=2 \times 10^{-5}$.

[Equipotential lines obtained with combination 2. $\Delta A^{\prime}=$ $2 \times 10^{-5}$.]

\section{Remarque :}

Afin de résoudre le problème posé avec une technique de pas à pas dans le temps, nous avons choisi une valeur initiale nulle dans tout le domaine ; les calculs ont montré qu'au bout d'une demipériode l'effet numérique transitoire impliqué par ce choix était terminé et que la solution périodique était atteinte.

\section{Conclusion.}

Nous avons exposé dans cet article deux types de méthodes mixtes en géométrie axisymétrique. En associant ainsi la méthode des éléments finis avec la méthode des éléments de frontière (couplage $n^{\circ} 1$ ) ou avec le développement d'une solution analytique (couplage $\mathrm{n}^{\circ} 2$ ) une meilleure modélisation du pro- 


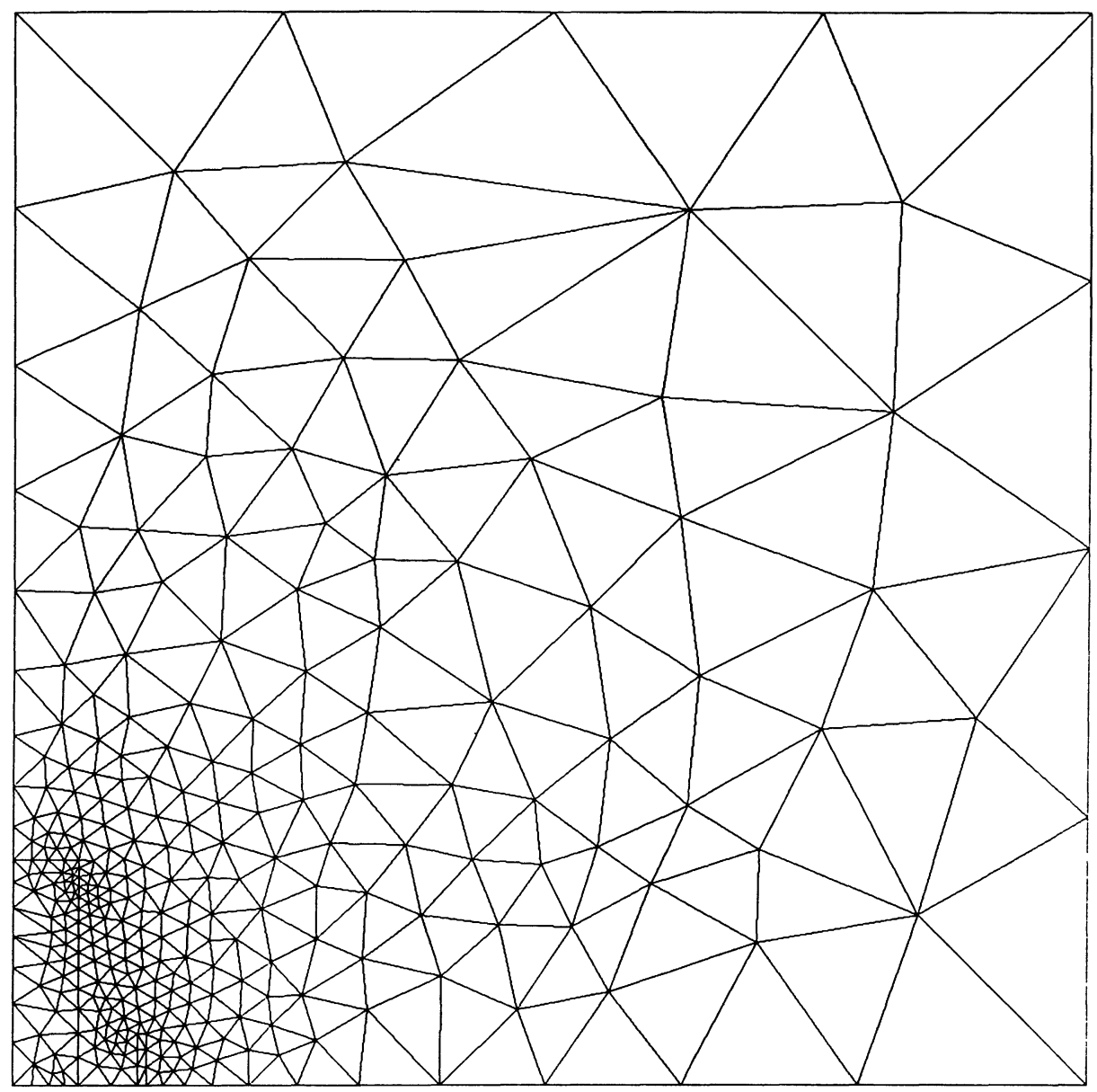

Fig. 7. - Maillage éléments finis de tout le domaine de calcul (éléments finis classiques).

[Finite element mesh of the entire solution domain (classical finite elements).]

blème physique est réalisée. Nous avons comparé ces deux types de couplage sur un même dispositif et utilisé ces modèles pour le calcul de courants de Foucault ; une bonne concordance avec des résultats expérimentaux a été obtenue. Il ressort de cette étude que le calcul de la matrice de rigidité extérieure pour le couplage $n^{\circ} 2$ est plus rapide que celui impliqué par le couplage $n^{\circ} 1$. Néanmoins le couplage $n^{\circ} 2$ qui conduit à un système linéaire d'ordre plus élevé possède globalement un temps de calcul environ deux fois plus long.

\section{Annexe}

Calcul de la dérivée normale de $\boldsymbol{A}^{\prime}$ sur la frontière.

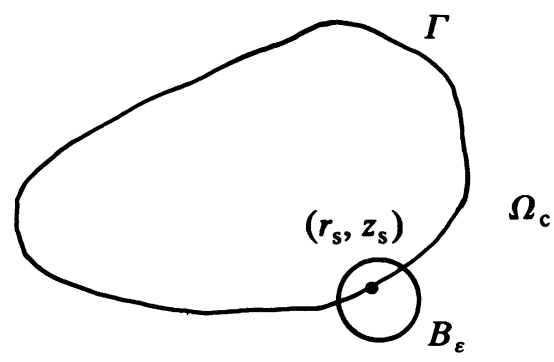

Appelons $\mathrm{B}_{\varepsilon}$ la boule de rayon $\varepsilon$ et centrée sur le point $\left(r_{\mathrm{s}}, z_{\mathrm{s}}\right)$. Pour toute fonction $\varphi^{\prime}$ à support borné défini dans $\mathbb{R}^{2}$ suffisamment continue et dérivable pour que toutes les intégrales aient un sens on peut écrire grâce à la formule de Green :

$$
\begin{aligned}
& \int_{\Omega_{\mathrm{c}}} \frac{1}{r_{\mathrm{s}}} \operatorname{grad} A^{\prime} \cdot \operatorname{grad} \varphi^{\prime} \mathrm{d} r \mathrm{~d} z= \\
& \quad=\int_{\Gamma} \varphi^{\prime} \frac{1}{r_{\mathrm{s}}} \frac{\partial A^{\prime}}{\partial n_{\mathrm{s}}} \mathrm{d} \gamma \\
& \quad=\int_{\Gamma} q \int_{\Omega_{\mathrm{c}}} \frac{1}{r_{\mathrm{s}}} \operatorname{grad} G \cdot \operatorname{grad} \varphi^{\prime} \mathrm{d} r \mathrm{~d} z \mathrm{~d} \gamma
\end{aligned}
$$

où $\mathbf{n}$ est la normale sortante du domaine $\boldsymbol{\Omega}_{\mathrm{c}}$. L'intégrale sur $\Omega_{\mathrm{c}}$ s'exprime :

$$
\begin{aligned}
& \int_{\Omega_{\mathrm{c}}} \frac{1}{r_{\mathrm{s}}} \operatorname{grad} G \cdot \operatorname{grad} \varphi^{\prime} \mathrm{d} r \mathrm{~d} z= \\
& \quad=\lim _{\varepsilon \rightarrow 0} \int_{\Omega_{\mathrm{c}} \backslash\left(\Omega_{\mathrm{c}} \cap B_{\varepsilon}\right)} \frac{1}{r_{\mathrm{s}}} \operatorname{grad} G \cdot \operatorname{grad} \varphi^{\prime} \mathrm{d} r \mathrm{~d} z .
\end{aligned}
$$


En employant de nouveau la formule de Green, (2) se transforme :

$$
\begin{aligned}
\int_{\Omega_{\mathrm{c}} \backslash\left(\Omega_{\mathrm{c}} \cap B_{\varepsilon}\right)} \frac{1}{r_{\mathrm{s}}} \operatorname{grad} G & \cdot \operatorname{grad} \varphi^{\prime} \mathrm{d} r \mathrm{~d} z= \\
= & \int_{\Gamma \backslash\left(\Gamma \cap B_{\varepsilon}\right)} \frac{1}{r_{\mathrm{s}}} \frac{\partial G}{\partial n_{\mathrm{s}}} \varphi^{\prime} \mathrm{d} r \mathrm{~d} z+ \\
& +\int_{\partial\left(B_{\varepsilon} \cap \Omega_{\mathrm{c}}\right)} \frac{1}{r_{\mathrm{s}}} \frac{\partial G}{\partial n_{\mathrm{s}}} \varphi^{\prime} \mathrm{d} \gamma
\end{aligned}
$$

Par passage à la limite il vient :

$$
\begin{aligned}
\int_{\Omega_{\mathrm{c}} \backslash\left(\Omega_{\mathrm{c}} \cap B_{\varepsilon}\right)} \frac{1}{r_{\mathrm{s}}} \operatorname{grad} G & \cdot \operatorname{grad} \varphi^{\prime} \mathrm{d} r \mathrm{~d} z= \\
= & \int_{\Gamma} \frac{1}{r_{\mathrm{s}}} \frac{\partial G}{\partial n_{\mathrm{s}}} \varphi^{\prime} \mathrm{d} \gamma+\frac{\varphi^{\prime}\left(r_{\mathrm{s}}, z_{\mathrm{s}}\right)}{2} .
\end{aligned}
$$

En reportant (2) dans (1) qui est vraie pour toute fonction $\varphi$ ' il vient l'égalité :

$$
\frac{1}{r_{\mathrm{s}}} \frac{\partial A^{\prime}}{\partial n_{\mathrm{s}}}=\frac{q\left(r_{\mathrm{s}}, z_{\mathrm{s}}\right)}{2}+\int_{\Gamma} \frac{1}{r_{\mathrm{s}}} \frac{\partial G}{\partial n_{\mathrm{s}}} q \mathrm{~d} \gamma
$$

où $\mathbf{n}_{\mathrm{s}}$ est la normale sortante de $\Omega_{\mathrm{c}}$ au point $\left(r_{\mathrm{s}}, z_{\mathrm{s}}\right)$.

\section{Bibliographie}

[1] Meunier G., Coulomb J. L., Krahenbul L., SALON S. J., Hybrid finite element solutions for three dimensional scalar potential problems I.E.E.E. MAG-22 (1986) 1040-1042.

[2] Huang Q. S., Krahenbuhl L., Nicolas A., Numerical calculation of steady-state skin effect problems in axisymmetry, I.E.E.E. Trans. Magn. MAG-24 (1988) 201-209.

[3] Salon J. L., Peng J. P., A hybrid finite elementboundary elements formulation of Poisson's equation for axisymmetric vector potential problems, J. Appl. Phys. 53 (1982) 8420-8422.

[4] Salon S. J., Schneider J. M., A hybrid finite element-boundary integral formulation of the eddy current problem, I.E.E.E. Trans. Magn. MAG-18 (1982) 461-466.

[5] Bouillault F., RAzEK A., Utilisation d'une formulation mixte (éléments finis-intégrale de frontière) pour modéliser les systèmes électromagnétiques, Revue Phys. Appl. 21 (1986) 595-601.
[6] CHARI M. V. K., Electromagnetic field computation of open boundary problems by a semi-analytic approach, I.E.E.E. Trans. Magn. MAG-23, n 5 (1987).

[7] Ren Z., Bouillault F., Razek A., Vérité J. C., Comparison of different boundary integral formulations when coupled with finite elements in three dimensions, I.E.E.-A. 135 (1988) 501-507.

[8] Abramovitz M., STEgun I. A., Handbook of Mathematical functions (Dover Publications) 1968.

[9] Tikhonov A. N., SAmarskil A. A., Equations of Mathematical Physics (Pergamon Press) 1963.

[10] Al-Shaikhli A. K. M., Hobson L., Novel technique for the design of induction billet heaters, I.E.E.B 133 (1986) 323-330.

[11] MortchelewiCZ G. D., Etude numérique d'un four à induction, Bull. DER-EDF $\mathrm{n}^{\circ} 1$ (1981) 45-65. 